\title{
EFFECT OF HYPERGLYCEMIA ON EPCS FUNCTION AND REGENERATIVE ABILITY
}

|||||||| كلية الآداب والعلوم

QATAR UNIVERSITY
Hadeel Hendawi ${ }^{1}$, Dina Awartani ${ }^{1}$, Aya Ghoul ${ }^{1}$

Supervisors: Haissam Abou Saleh ${ }^{1}$, Isra Marei ${ }^{2}$, Christopher Triggle ${ }^{2}$

Undergraduate Students, Biomedical and Health.

${ }^{1}$ Qatar University, ${ }^{2}$ Weill Cornell Medicine Qatar

(a)

Weill Cornell

Medicine-Qatar

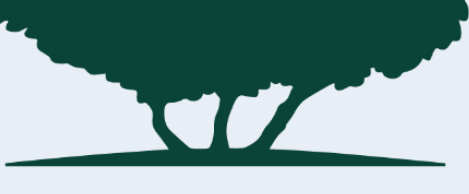

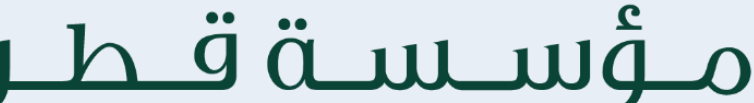
Qatar Foundation

\section{Introduction}

Diabetes is characterized by elevated glucose levels in the blood or "hyperglycemic conditions" due to the defect in insulin action or secretion. These conditions increase the risk of cardiovascular complications as they impact vascular endothelial cells causing vascular dysfunction. Vascular endothelium repair occurs by resident endothelial cells, however these cells have limited proliferation and self-repair ability. It has been suggested that endothelial progenitor cells (EPCs) participate in the repair and maintenance of vascular endothelial cells as well as assisting in the generation of blood vessels. EPCs have been classified into two different subtypes according to their emergence in culture; early EPCs and late EPCs (or Blood outgrowth endothelial cells, BOECs); and these types differ in their origin, phenotype and differentiation abilities. While early EPCs are broadly studied in diabetes, the role of late EPCS/BOECs and the mechanisms of their repair are not clear. Late EPCs/BOECs are known to have potential regenerative abilities and might constitute a potential cure to protect form cardiovascular complications in diabetic patients. This study aims to view the side effects of hyperglycemic environment on BOECs viability, cytotoxicity, and regenerative functions.

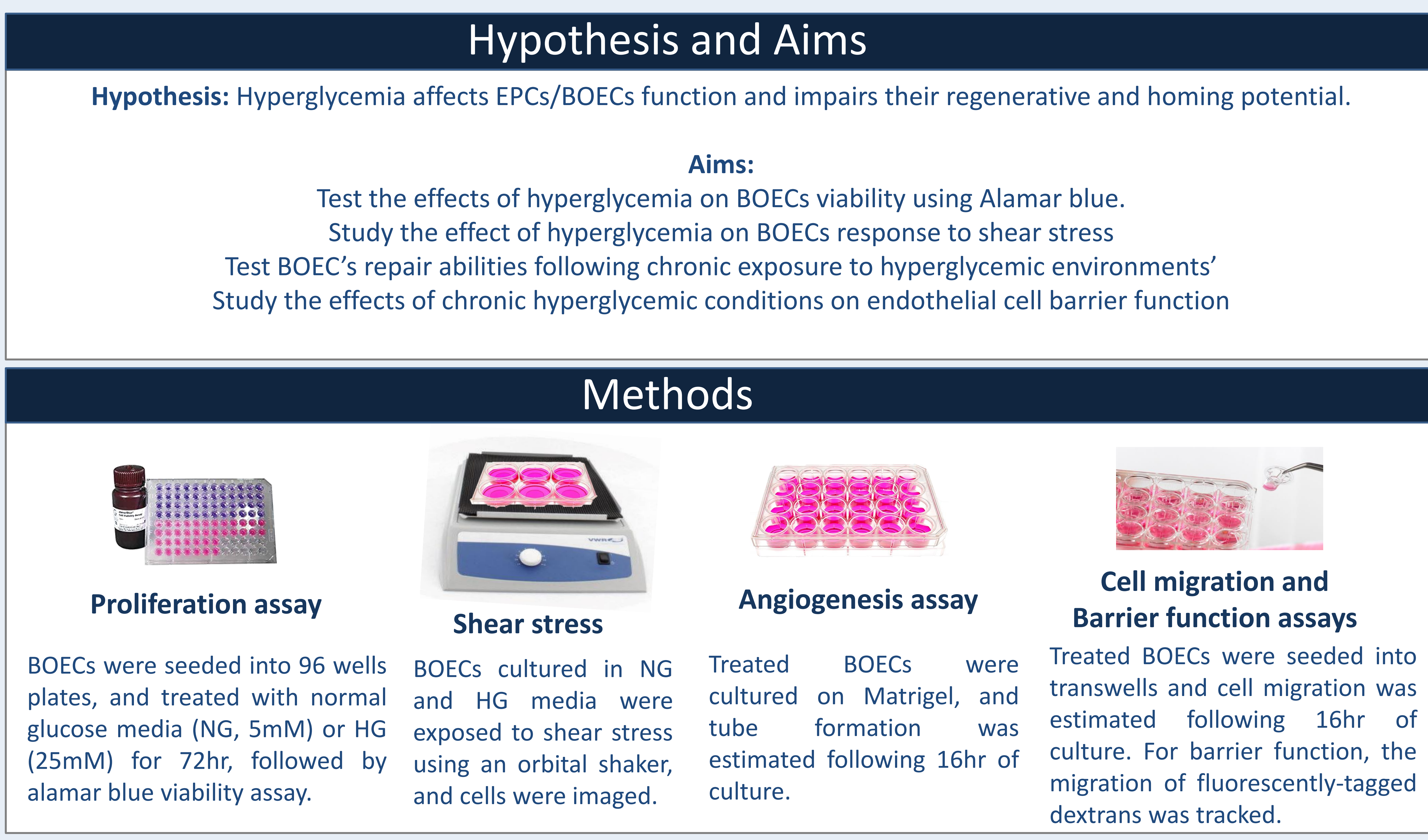

\section{Results}

- Exposing BOECs to HG media for 3 days resulted in a significant reduction in viability (Fig 1)

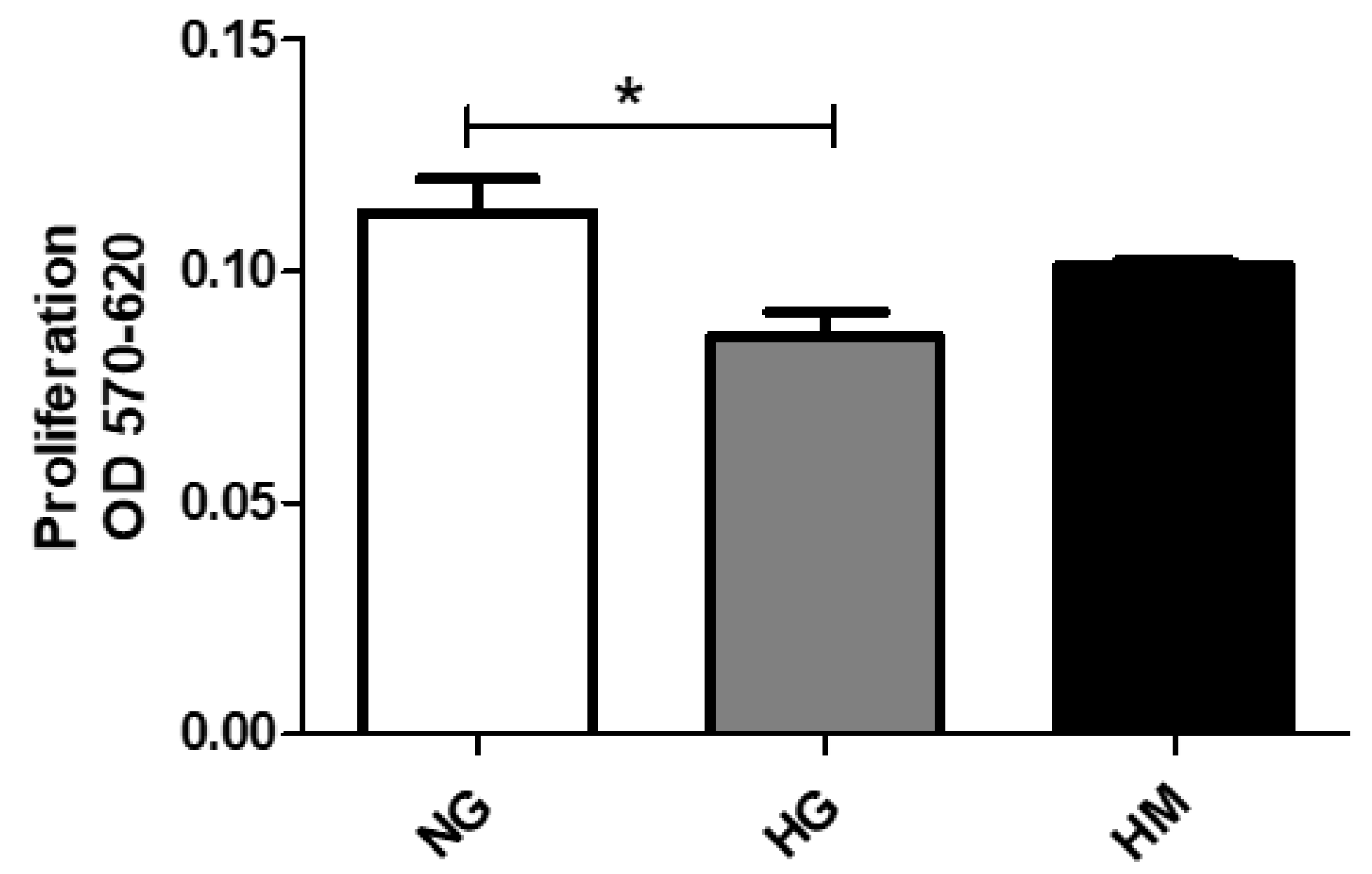

Figure 1. Cell viability assay measured as the reduction in alarm blue stain Exposure of BOECs to HG reduced viability, while using mannitol control didn't affect proliferation. $n=3$ using one BOEC isolate. Analysis was done using one way ANOVA. ${ }^{*} P<0.05$

- Cell alignment and elongation under hyperglycemic conditions in response to shear stress NG

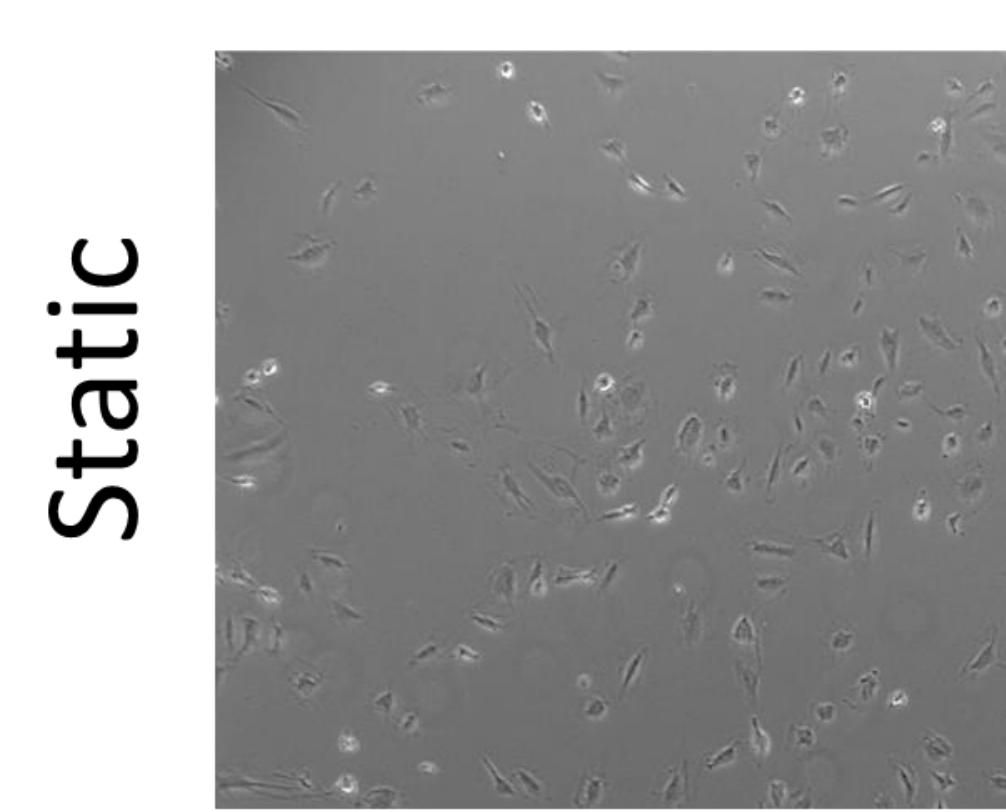

\section{(1)}

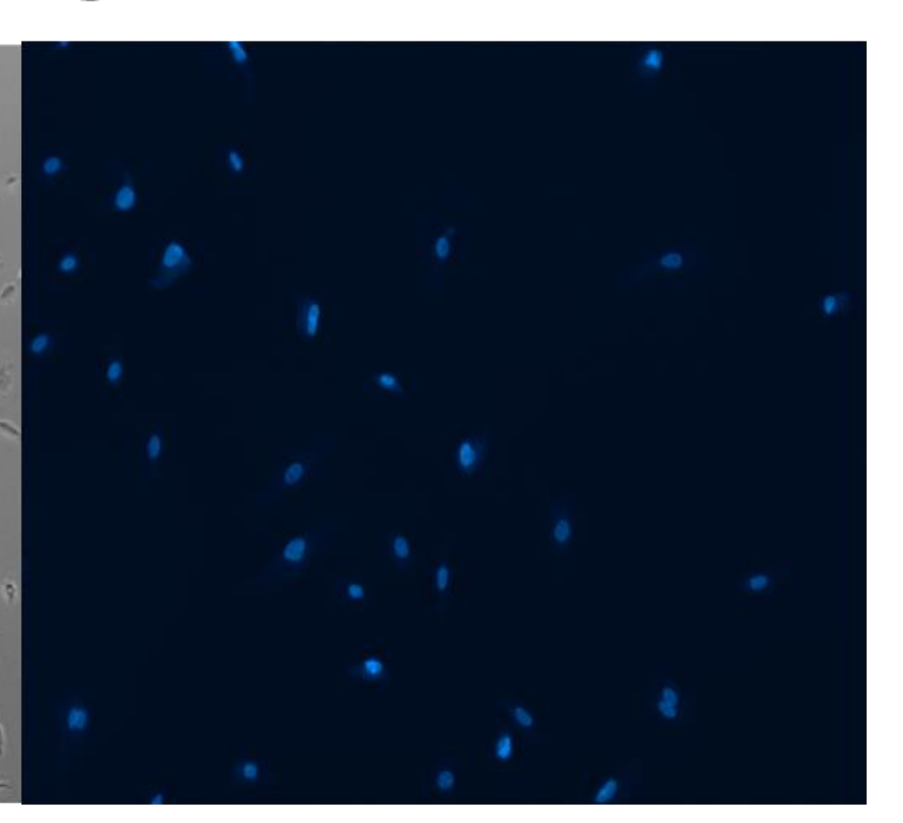

맘

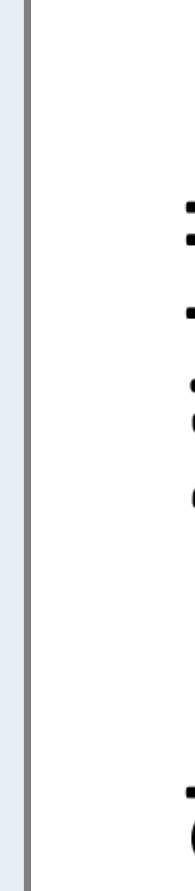
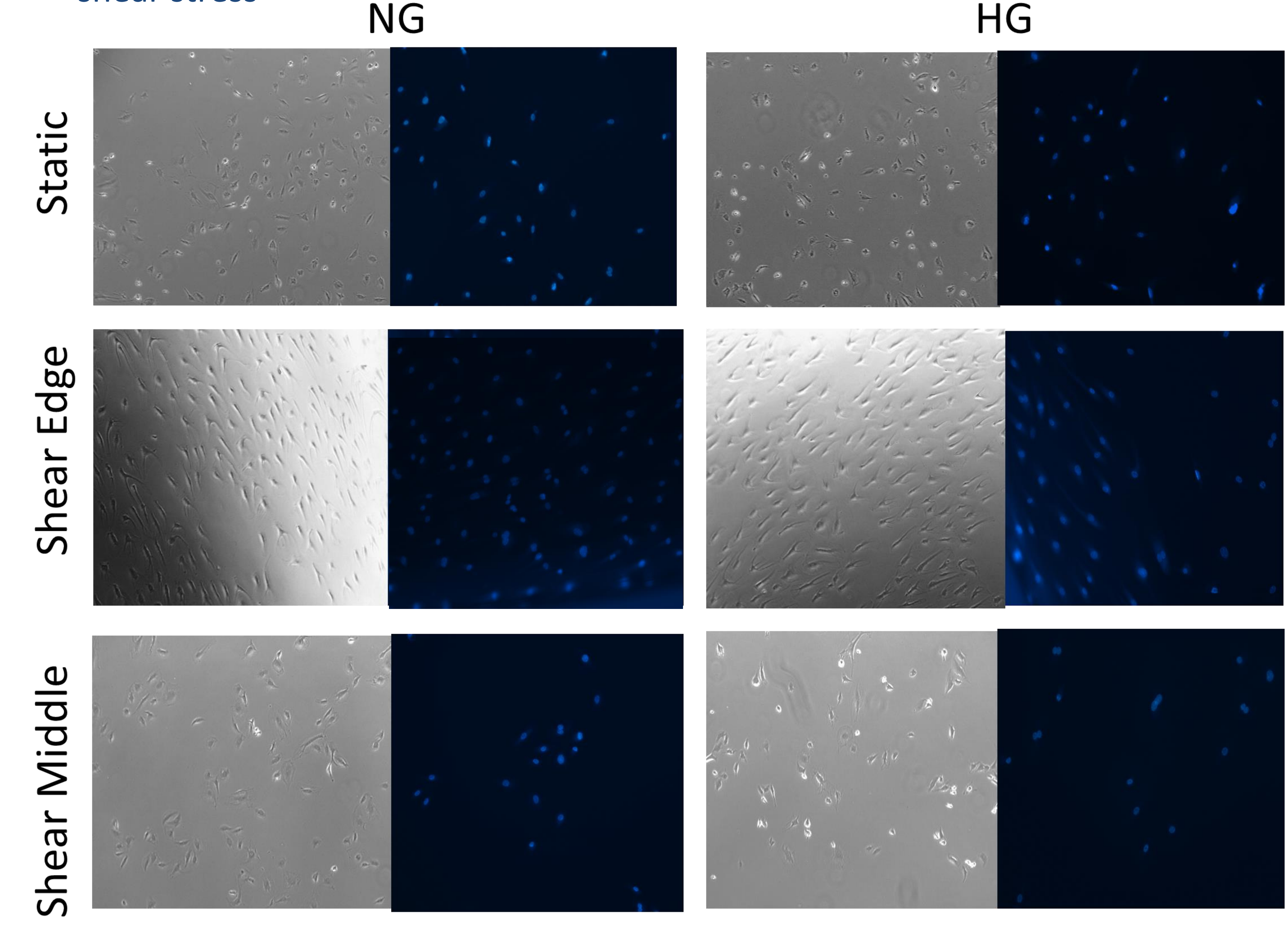

Figure 2. Cell alignment and morphology under shear stress for NG vs HG under light microscope and using DAPI stain. The cells on the edges were under unidirectional stress in the orbital shaker while the center was left disturbed and the figure clearly shows the difference in cells morphology under both conditions.
- Exposing BOECs to HG media for 3 days didn't affect tube formation. However, the mean mish size was affected in HG treated cells.
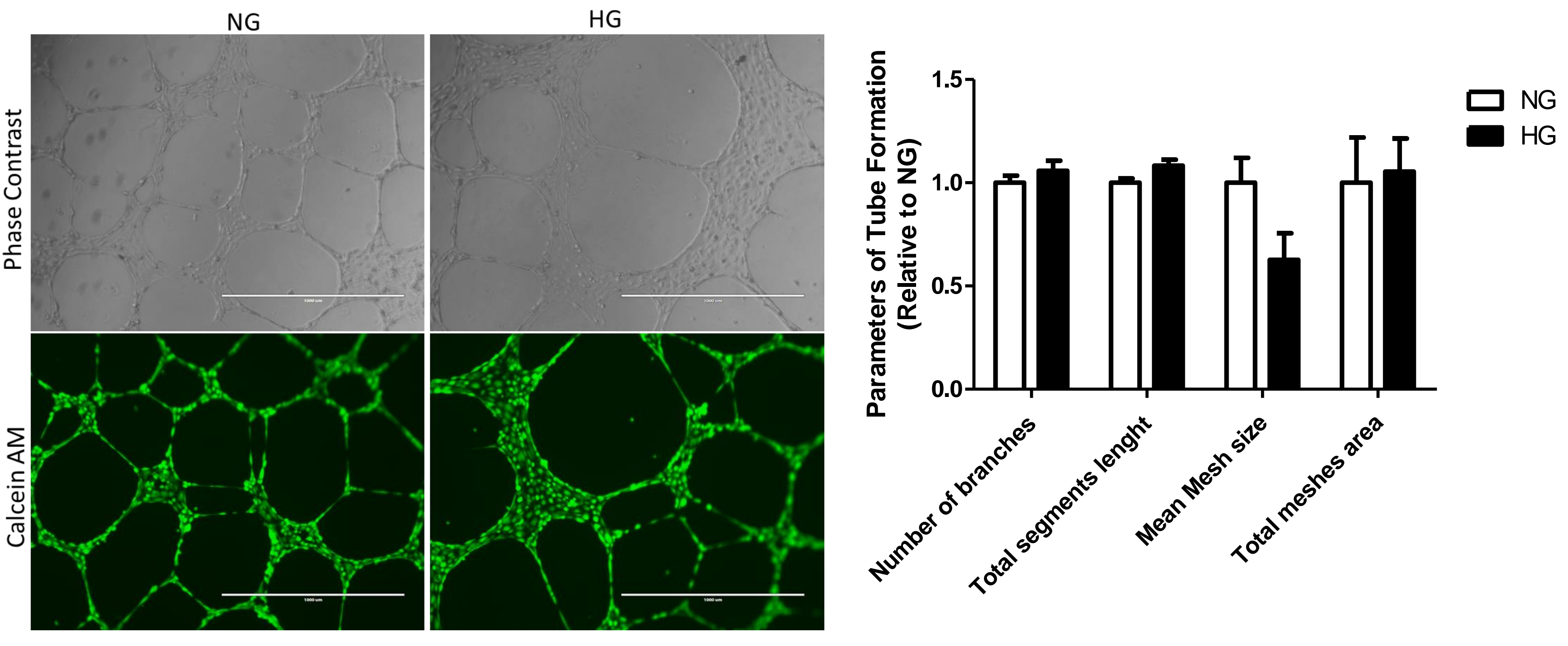

Figure 3. Tube formation assay measured in Matrigel. BOECs treatment with $H G$ media for 3 days didn't affect tube formation. Tube formation parameters were corrected to NG. $n=3$ using one BOEC isolate. Analysis was done using one way ANOVA. ${ }^{*} P<0.05$.

- Exposing BOECs to HG media for 3 and 6 days significantly reduced BOECs migration ability through transwells.
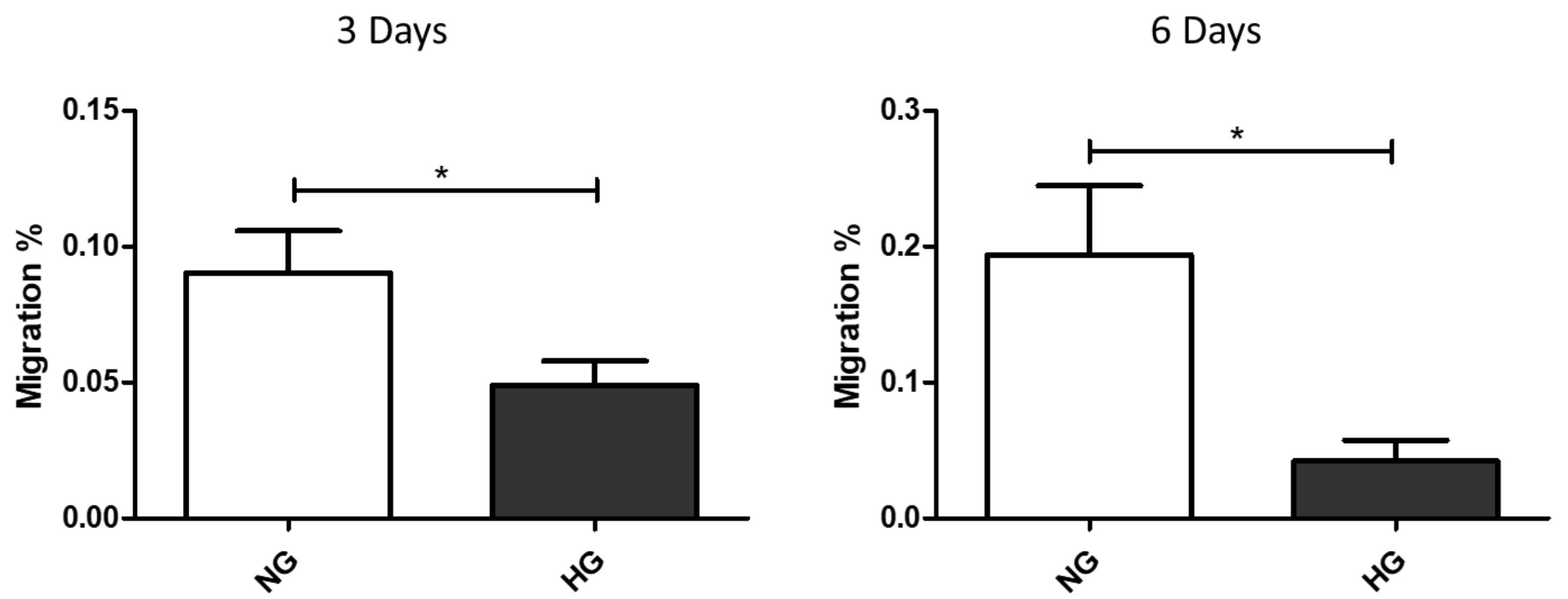

Figure 4. Cell migration assay using transwell system. Migration of BOECs treated with $H G$ for 3 and 6 days was significantly reduced. $n=3$ using one BOEC isolate. Analysis was done using t-test. ${ }^{*} P<0.05$.

- Exposing BOECs to HG media for 3 days resulted in increased leakage of 150 and 20KDa dextrans; however these differences were not significant.
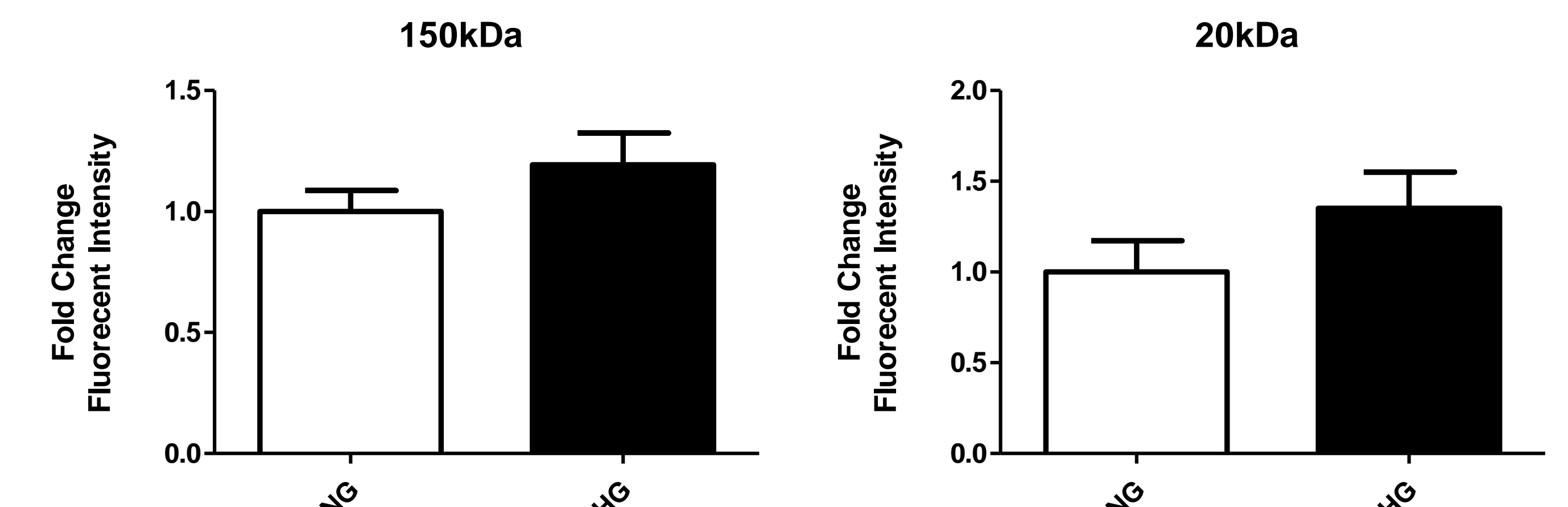

Figure 5. Barrier function as measured by the leakage of dextrans through cell monolayers cultured on transwell systems. $n=3$ using one BOEC isolate. Analysis was done using t-test.

\section{Conclusion}

In conclusion, hyperglycemia causes a plethora of negative effects on the endothelial cells, it decreases cell migration and barrier function. These functions are vital and are strongly depended on for the regeneration of the blood vessels as well as the control of the exchange of nutrients and waste between blood and tissues, and protection form pathogens. These effects are consistent with some symptoms shown by diabetic patients with cardiovascular complications. The future prospects for this area of research is to investigate the pathways of cell signaling involved in the vasculogenesis and angiogenesis in order to find ways to reverse or reduce the effects of

\section{References:}

Analysis of Endothelial Barrier Function In Vitro. Vol: 763 Humana Press. 2011

Mechanisms of endothelial cell migration. Vol: 71. Cell. Mol. Life Sci. 2014

Blood cells and endothelial barrier function. Vol: 3. Tissue 